\title{
Advanced raman spectroscopy in nanomedicine
}

\author{
Volume 3 Issue 3 - 2016
}

\section{Editorial}

Abbreviations:SERS, Surface-Enhanced Raman Scattering; TERS, Tip-Enhanced Raman Scattering; SPM, Scanning Probe Microscopy; TPL, Two-Photon Luminescence; CARS, Coherent AntiStokes Raman Scattering; SRS, Stimulated Raman Scattering

\section{Introduction}

Optical microscopy is a key technique to biomedical research. Light microscopy reveals changes inrefractive index within cells and tissue, however lacks of biochemical contrast and is only appropriate

for monolayers of cells or tissue slices and can produce only the image of the surface or optical contrast in the case of inverted microscopy. Fluorescence imaging is the current major technique in biomedical research. It is based on the labeling of the molecules by fluorescent dyes. The fluorescence can be expressed by modified genes or labelled molecules under investigation. The fluorescence imaging have a number of drawbacks:

a. sample modification by labeling;

b. label can perturb the behaviour of the tagged molecule of interest;

c. photobleaching limits the observation time.

The Raman scattering phenomenon known for more than 80 years, during the last decades is gaining more and more attention in analytical applications and can provide chemical finger prints of cells, tissues or biofluids. In contrast to established analytical techniques, Raman spectroscopy provides label-free, non-destructive, chemically selective and spatially resolved analysis. The high chemical specificity, simple sample preparation procedure and the ability to use advanced optical technologies in the visible or nearinfrared spectral range have recently led to an increase in medical diagnostic applications of Raman spectroscopy. The basic idea of the application of Raman spectroscopy in medicine is the hypotesis that deseases effect the molecular structure of cells and can be detected and quantified by Raman spectroscopy. Based on advanced technical development, Raman spectroscopy can be implemented into diverse setups ranging from confocal microscopes for acquisition of threedimensional spectral information up to hand-held fiber devices for direct use in clinical diagnostics. Furthermore, recent progress in the field of multivariate data analysis allows for processing such complex spectral data into scientific information for fundamental research as well as for patients to obtain objective data for diagnosis. ${ }^{1}$ The new strategies have been developed to overcome the weak signal problems of Raman spectroscopy by application of non-linear optics and localized nanoplasmonic effects to enhance the Raman signals to increase the acquisition speed and accuracy of diagnosis. Application of nanotechnology solutions in Raman spectroscopy and microscopy, like SERS and TERS, open a new directions in Nanomedicine, based on a single molecule science. ${ }^{2}$

\section{Physiological investigations of tissues and cells}

Raman micro-spectroscopy provides an easy to use, non-

\author{
Valentinas Snitka \\ Kaunas University of Technology, Lithuania
}

\begin{abstract}
Correspondence: Valentinas Snitka, Research Center for Microsystems and Nanotechnology, Kaunas University of Technology, Student str. 65, Kaunas, Lithuania, Tel 3703745I 588 Email vsnitka@ktu.lt
\end{abstract}

Received: February 16, 2016 | Published: March 17, 2016

destructive, and spectra based imaging tool of probing cell and tissue physiology with diffraction-limited resolution, however in combination with Scanning Probe Microscopy (SPM) the spatial resolution up to 10 nanometers can be achieved. ${ }^{3}$ By probing the vibrational signature of molecules and molecular groups, the distribution and metabolic products of molecular activity that cannot be imaged using fluorescent dyes can be investigated. The non destructive imaging and characterization of single cells by Raman spectroscopy was demonstrated by several authors. ${ }^{4}$ Authors managed to obtain the Raman spectra from the different organelles of the cells and from isolated chromosomes by micro-Raman spectroscopy with diffraction limited resolution. Since then, Raman spectroscopy has been applied to study the physiology of a wide range of cells, bacteria and viruses, as well as plant cells, and the interaction of drug molecules, nanoparticles and other molecules with cells. ${ }^{5-6}$

\section{Drug-cell interactions}

The focus for the development of novel therapeutics is moving from classical solid dosage systems such as tablets and capsules to modern drug carriersystems in micro/ nanometer size range. In this tendency, an appropriate analytical analysis and evaluation of interactions of drug delivery systems, their cellular uptake of drugs and nanoparticles and the release mechanism, the kinetics for an incorporated drug in order to improve therapeutics and specific disease treatment protocols are of high importance. The Raman spectroscopy as non-invasive, chemically sensitive analytical technique is openning a new ways in the field. ${ }^{7-9}$

\section{Raman imaging of cells and drug delivery systems}

Raman spectral imaging is a method for producing chemical images based on Raman spectra collected during the sample scanning procedure. A complete spectrum is acquired at each and every pixel of the image, and then used to generate a colour images based on biomaterial composition and structure. A typical experiment uses $\mathrm{X}-\mathrm{Y}$ sample scanning line by line and spectrum acquisition, repeated many times (up to thousands) to collect data from the area of interest. Raman spectral images can be collected in 2D and 3D dimensions, to produce $\mathrm{XY}$ images, $\mathrm{XZ}$ and $\mathrm{YZ}$ slices, and $\mathrm{XYZ}$ data hyperspectra files. The technical progress during the last years led to an increased accessibility and a variety of available instruments on the market. ${ }^{10-13}$ Although components and exhibited surface structures varied broadly in pharmaceuticals, images with high spatial resolution and good 
chemicals electivity were demonstrated in the work. ${ }^{14}$ The resolution of the cellular Raman image is an important factor to select a different molecular response and to capture the subcellular structures up to nanometer size range as well as the nano carriers. The lateral resolution depends on the numerical aperture of the objective in combination with the wave length of the exitation laser light, and the axial resolution depends on the pinhole diameter and the refractive index of the environment. ${ }^{15}$ The currently available Raman instruments, produce a lateral resolution down to $200 \mathrm{~nm}$ and anaxial resolution of $500 \mathrm{~nm}$ can be achieved. It makes the Raman microscopy technique feasible for visualization of subcellular structures and drug carriers. ${ }^{16-17}$

\section{SERS and TERS}

SERS opens a new possibility to dramatically increase the sensitivity of Raman spectroscopy in a wide range of systems and to move to one molecule science and applications. Combining the microscopic technology with optical measurements, single-molecule Raman has been established as a significant spectroscopic method to probe single molecules, exploring the high sensitivity and spectral resolution of SERS. ${ }^{18}$ and TERS. ${ }^{19}$ The SERS nanosensors can be used in various applications like $\mathrm{pH}$ sensing, detection of drugs and proteins, toxins, and diagnostic of genes, finger printing of cells. For DNA detection the SERS based nanoprobe can be used as a bioassay in solution or on a chip platform. Gold nanoparticles provide a complex theranostic platform, combining Raman and SERS with luminescence imaging as well as photodynamic therapy and photothermal therapy. Plasmonicsstimulated and optically modulated delivery of nanoparticles into brain tumor in live animals was demonstrated and gold nanoparticle based photothermal treatment of tumor vasculature may stimulate inflammasomes activity, thus increasing the permeability of the blood brain barrier. The imaging method using TPL of gold nanoparticles provides an unprecedented spatial selectivity for targeted nanoparticles delivery to tumor tissue.$^{20}$

\section{Beyond the classics}

Thelow Raman scattering cross section of molecules is the major drawback of spontaneous Raman microscopy limiting the application in biomedicine. Therefore, Raman microscopy is a slow technique, with long acquisition time, especially in biological fields. The progress towards faster imaging recently have been made, however the long imaging time still is a limiting factor for wider applications. The auto fluorescence, which is present in many biological samples, is an other limiting factor of ten prevents the detection of a Raman signal. In order to solve the problems caused by these limitations, the Raman techniques based on non-linear optical microscopy, namely CARS and SRS microscopy have been developed during the last years. After an initial period of the development of methodological issues, both schemes have found a lot of applications during the recent years. Recently published review articles give an excellent overview over the current state-of-the-art of biomedical applications of CARS and SRS microscopy. ${ }^{21}$

\section{Is Raman Spectroscopy Ready for Clinical Use?}

Recent reports and experts opinions. ${ }^{22}$ demonstrate a fast growing increase in the development and application of Raman spectroscopy based methodologies to address biomedical questions. The label-free ability to obtain specific chemical information about the biological molecules and tissues, makes Raman spectroscopy an attractive analytical tool in clinical diagnostics of cells, tissue biopsies and pathogens. The significant progress has been made towards the application of micro-Raman spectroscopy as a standard test for the fast identification of pathogens and the determination of the pathogens resistance to antibiotics. The Raman spectromicroscopy, as a labelfree and video-rate imaging technique may have a tremendous impact on identifying a cancer tumor tissue or other abnormalities. It seems a few years from now we will see the first Raman applications as standard diagnostic or therapeutic tool in a daily clinical practice.

\section{Acknowledgments}

None.

\section{Conflicts of interest}

None.

\section{References}

1. Popp J, Windbergs $M$ Pharmaceutical applications of Raman spectroscopy-From diagnosis to therapeutics. Adv Drug Deliv Rev. 2015;89:1-2.

2. Chen J, Ding W, LuoZ Probing single molecules and molecular aggregates: Raman spectroscopic advances. J Raman Spectroscopy. 2015

3. Bohme R, Richter M, Cialla D et al. Towards a specific characterisation of components on a cell surface - combined TERS-investigations of lipids and human cells. J Raman Spectrosc. 2009;40(10):1452-1457.

4. Puppels GJ, Mul FFM, Otto C et al. Studying single living cells and chromosomes by confocal Raman microspectroscopy. Nature. 1990;347(6290):301-303.

5. Kong K, Kendall C, Stone N et al. Raman spectroscopy for medical diagnostics - From in-vitro biofluid assays to in-vivo cancer detection. Adv Drug Deliv Rev. 2015;89:121-134.

6. Schie IW, Huser T Label-free analysis of cellular biochemistry by Raman spectroscopy and microscopy. Compr Physiol. 2013;3(2):941-956.

7. Romero G, Qiu Y, Murray RA et al. Study of intracellular delivery of doxorubicin from poly(lactide-co-glycolide) nanoparticles by means of fluorescence lifetime imaging and confocal raman microscopy. Macromol Biosci. 2013;13(2):234-241.

8. Zukiene R, Snitka V Zinc oxide nanoparticle and bovine serum albumin interaction and influence on cytotoxicity in vitro. Colloids Surf B Biointerfaces. 2015;135:316-323.

9. Farhane Z, Bonnier F, Casey A et al. Raman micro spectroscopy for in vitro drug screening: subcellular localisation and interactions of doxorubicin. Analyst. 2015;140:4212-4223.

10. http://www.horiba.com/scientific/products/raman-spectroscopy/ramanimaging/

11. https://www.bruker.com/de/products/infrared-near-infrared-andraman-spectroscopy/raman.html

12. http://www.witec.de/products/raman/

13. http://www.ntmdt.com/afm-raman/ntegra-spectra

14. Kann B, Windbergs M Chemical Imaging of Drug Delivery Systems with Structured Surfaces-a CombinedAnalytical Approach of Confocal Raman Microscopy and Optical Profilometry. AAPS J. 2013;15(2):505-510.

15. Breuninger S, Henrich $\mathrm{M}$, Dieing $\mathrm{T}$ Key Elements of Confocal Raman Microscopy for High-Resolution Imaging. Spectroscopy. 2014;29(6):1-4.

16. Batiuskaite D, Grinceviciute N, Snitka V Impact of graphene oxide on viability of Chinese hamster ovary and mouse hepatoma $\mathrm{MH}-22 \mathrm{~A}$ cells. Toxicol In Vitro. 2015;29(5):1195-1200. 
17. Ma X, Qu Q, Zhao Y et al. Graphene oxide wrapped gold nanoparticles for intracellular Raman imaging and drug delivery. J Mater Chem B. 2013;1:6495-6500.

18. Wu D, Li JF, Ren B et al. Electrochemical surface-enhanced Raman spectroscopy of nanostructures. Chem Soc Rev. 2008;37(5):1025-1041.

19. Sharma G, Deckert-GaudigT, Deckert V Tip-enhanced Raman scattering-Targeting structure-specific surface characterization for biomedical samples. Adv Drug Deliv Rev. 2015;89:42-56.
20. Vo-Ding T, Yang Liu Y, Fales AM et al. SERS Nanosensors and Nanoreporters: Golden Opportunities in Biomedical Applications. Nanomed Nanobiotechnol. 2015;7(1):17-33.

21. Winterhalder MJ, Zumbusch A Beyond the borders- Biomedical applications of non-linear Raman microscopy. Adv Drug Deliv Rev. 2015;89:135-144.

22. L'Heureux M Analysis of the State of the Art: Raman Spectroscopy. Spectroscopy. 2015;30(6):1-4. 